\title{
ON CONFIGURATION-BASED OPTIMAL DESIGN OF LOAD-CARRYING LIGHTWEIGHT PARTS
}

\author{
Harl, B.; Predan, J.; Gubeljak, N. \& Kegl, M. \\ Faculty of Mechanical Engineering, University of Maribor, Smetanova 17, 2000 Maribor, Slovenia \\ E-Mail: bostjan.harl@um.si,jozef.predan@um.si,nenad.gubeljak@um.si,marko.kegl@um.si
}

\begin{abstract}
This paper discusses some aspects of FEA-based design procedures driven by topology optimization methods. Within this scope the problem of ensuring the robustness and reliability of optimized loadcarrying lightweight parts is addressed. To achieve this goal the engagement of the so called design configuration with subsequent topology optimization is proposed. Adequate configuration tools can be used to define a shell/lattice structure and subsequent topology optimization makes sure to remove the stress concentrations and lower the stress levels to a minimum. The numerical and experimental results of the presented example illustrate the importance of proper cell type and orientation selection, as well the importance of optimization, especially, if the underlying part is planned to be produced by 3D printing.
\end{abstract}

(Received in May 2016, accepted in January 2017. This paper was with the authors 3 months for 1 revision.)

Key Words: Load-Carrying Part, Lightweight Design, Topology Optimization, Lattice Configuration

\section{INTRODUCTION}

Load-carrying parts are the most fundamental constituents of practically all engineering structures, ranging from buildings to all kinds of machinery or apparatus. As the name implies, the main purpose of these parts is to ensure the intended functionality of the system by carrying loads and resisting to all possible mechanical impacts. In the past, the loadcarrying capability was by far the most important property of such a part. Nowadays, however, the increased competition and the cost-reduction demands are shifting this importance also to other properties. From the engineering point of view, perhaps two of such most exposed properties are: (a) prolonged lifespan, and (b) reduced material consumption (lightweight).

For a load-carrying part, a combined request for prolonged lifespan and lightweight mainly translates to reduced stress levels and removed stress concentrations at low material consumption. In a general case, this can hardly be done by engaging only experience and intuition. Therefore, numerical stress analysis procedures, such as the finite element analysis (FEA), are here of extreme importance in order to assure good results. Roughly speaking, until recently, a typical design cycle typically involved a design proposal with a subsequent stress analysis in order to check the ability of the part to resist the imposed loads. The stress analysis results were then used to improve the design for another design cycle. Nowadays, however, this is beginning to change due to the impressive development of numerical topology optimization methods, driven by underlying full FEA procedures [1].

In general, a FEA-based topology optimization process generates a design with the lowest stress levels with respect to the available/desired material volume used for the part. Theoretically, this means that conventional stress-checking design cycles are not needed any more, because an automated numerical optimization process is able to generate the best possible design - a lightweight structure with the lowest stress levels attainable.

Due to the immense number of excellent publications, see e.g. [2-7], related to new topology optimization methods one might get the impression that the low-stress design process is well resolved and automated. This, however, is not reflected in practice because 
practical engineering implementation limps far behind current development of the optimization methods. Although the reasons for this are quite diverse, by far the most important ones are typically related to the fact that successful topology optimization simply requests a much more precise modelling of boundary conditions (description of structural loads and supports) than this is commonly accepted in a usual FEA procedure. To lower the difficulty of a considered problem, engineers that are trained for ordinary FEA, frequently engage modelling simplifications, which are perfectly valid in this scope. In the scope of topology optimization, however, many of such simplifications are not allowed anymore, because they may lead to bad or even very wrong results. What makes the situation even worse is a simple fact that bad topology optimization designs might not be easily recognized because they might look quite reasonable and might exhibit acceptable stress levels for the specified loads.

In the quest to reduce the risk of getting bad results and maybe even not recognizing this, one has to think how to make the results less sensitive to variations in structural boundary conditions, see e.g. $[8,9]$. This paper focusses on one possible direction how to achieve this. Inspired by the observations from the field of bionics, the underlying idea is to force the topology optimization process to generate designs closer to efficient structural solutions found in nature. For this purpose a special procedure, here termed 'design configuring' is used.

A very important point to be kept in mind is that 'forcing' a topology optimization process into something, e.g. by restricting the available design space, will result in designs that are not fully optimal in the mathematical sense. However, one has to remember that the actual part will not be engaged in a strict mathematically described environment. The actual environment will be different with many unexpected circumstances (boundary condition variations). Therefore, a 'forced' topology optimization design might turn out to be more optimal and reliable in an actual situation.

The outline of this paper is follows. Section 2 presents briefly the underlying ideas and concepts in topology optimization. Section 3 discusses and compares the conventional and configuration-based topology optimization. Section 4 presents a numerical example problem and the numerically obtained design. Section 5 compares the numerical results with experiment and analyses thoroughly the data with respect to expected results and behaviour.

\section{TOPOLOGY OPTIMIZATION ESSENTIALS}

FEA-based topology optimization methods come in a rather large variety, but they mostly share a few basic ideas and concepts, which constitute their foundation. Roughly, these ideas can be described as follows.

In order to control the presence or absence of material in individual regions of the design space, typically a set of design parameters $p_{i}$ is introduced. Usually, $p_{i}$ is a dimensionless parameter in the range $[0,1]$, sometimes also termed the 'numerical material density'. It is used to define computational values of material properties such as density and modulus of elasticity. Typical relations are similar to

$$
\rho_{i}=p_{i} \rho_{0}, E_{i}=p_{i} E_{0}
$$

where $\rho_{0}$ and $E_{0}$ are the actual density and elastic modulus of the used material, while $\rho_{i}$ and $E_{i}$ are the corresponding computational values used for the FEA. In this way a certain region of the design space tends to become void as $p_{i} \rightarrow 0$ or tends to become full material as $p_{i} \rightarrow 1$.

A particular design parameter $p_{i}$ is typically associated with either: (a) a particular finite element, or (b) a particular finite element node. In either case the whole set of design parameters $p_{i}$ defines current material distribution in the whole design space, which, in turn, is defined by the finite element mesh. 
Once the design parameters are well defined, one can proceed with the formulation of the optimization problem, most importantly, by the definition of the objective function to be minimized. In conventional topology optimization of statically loaded structures, the objective function $f$ is typically defined as the total strain energy of the structure. So, in the case of linear elasticity, the objective function (total strain energy) can be written as

$$
f=\frac{1}{2} \int_{B} \boldsymbol{\sigma}: \varepsilon d V
$$

where $\boldsymbol{\sigma}$ denotes the Cauchy stress tensor, $\boldsymbol{\varepsilon}$ is the small-strain tensor, and integration has to be done over the whole domain (volume) $B$ of the structure. Note that using the small-strain theory is usually a very good choice, even if the actual response of a part involves moderate geometrical nonlinearities. This is because small-strain theory ensures high stiffness in the initial configuration, which is often the most important requirement even under a moderately nonlinear response.

If the objective function is defined in this way, the optimizer will redistribute the material within the design domain in such a way that the total strain energy will be minimized. Such a minimization of the strain energy has some highly desirable collateral effects, typically sought by engineers. Most of all, if a given or prescribed material quantity is redistributed by strain energy minimization, then:

- Stress concentrations will vanish on all cut surfaces, where the term 'cut surface' is used to denote the material/void interface within the finite element mesh.

- The stress levels will reach at least near minimal values attainable.

- The overall structural stiffness will be maximized.

All of these are typically highly desirable features of a load-carrying part. Namely, high stiffness is usually preferable because machinery can operate better and more precisely in such a case, see e.g. [10]. On the other hand, removed stress concentrations and lower stress levels drastically reduce the risk of material fatigue cracks to appear. This will prolong the service life of a part dramatically and substantially reduce the possibility of structural failure.

An important and desirable, but potentially dangerous, effect of topology optimization is that the final design is fully adapted to given boundary conditions (loads and supports). This means that a good design can only be obtained, if all possible loading conditions are taken into account during optimization. An individual loading situation that may be applied to the structure at some given time is termed a 'load case'. Thus, handling of multiple load cases is an unavoidable necessity, and is usually implemented by applying the MAX operator in the following way.

Let $f_{i}$ denote the strain energy of the $i^{\text {th }}$ region, influenced by the design parameter $p_{i}$, within the design domain. Note that in dependence on the definition of $p_{i}$, the $i^{\text {th }}$ region can be either a finite element or a FE node surrounding. In this case the objective function, corresponding to a single-load-case-situation, can be written as

$$
f=\sum_{i} f_{i}
$$

In case of multiple load cases, all corresponding strain energies have to be addressed. Let $f_{i}^{k}$ denote the strain energy of the $i^{\text {th }}$ region, corresponding to the $k^{\text {th }}$ load case. In such a situation the actual objective function, to be minimized, is typically defined as

$$
f=\sum_{i}\left(\max _{k} f_{i}^{k}\right)
$$

The minimization of such an objective function will yield the best possible design with respect to all considered load cases. 


\section{CONVENTIONAL VERSUS CONFIGURATION-BASED OPTIMIZATION}

Conventional topology optimization can be regarded as a task consisting of two sequential steps as follows:

a) define the optimization domain;

b) distribute optimally the available material within the whole domain.

Within this scope the optimizer is allowed to redistribute the material within the whole design space without any limitations. The result should be the best possible result adapted precisely to the considered load cases. What is worrying here is that one cannot know how the design will respond to any unexpected load case variations.

To mitigate these concerns, a potentially interesting direction might be to get help from the field of bionics, e.g. [11, 12]. From nature we know that mechanically very efficient biologic structures are often what we call shell or lattice structures. Keeping this in mind it is reasonable to expect that more robust designs could potentially be obtained by forcing the topology optimizer to generate such kind of structures. This can actually be achieved by expanding the two-step optimization task into a three steps one, as follows:

a) define the optimization domain;

b) configure the optimization domain by enforcing a shell/lattice structure;

c) distribute optimally the available material within the configured domain.

Fig. 1 illustrates a structural part, whose design domain consists of two equal symmetric regions [13]. The left region (solid design; green colour) is left untouched and is ready to be optimized conventionally. On the other side, the right region (lattice design; blue colour) was configured as a lattice structure and is ready to be optimized within the design space limits, imposed by the lattice configuration. It might be worth noting that a lattice structure is enforced on a volume domain defined by the FE mesh. On the other hand, a shell configuration is enforced on a surface domain, defined by the outer hull of a FE mesh.

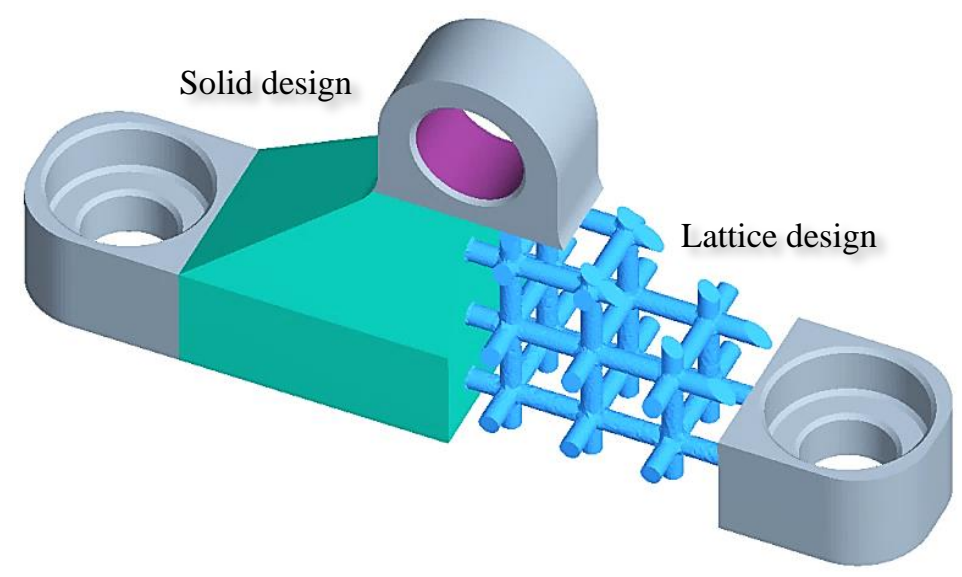

Figure 1: A part with two equal symmetric optimization regions: left one is untouched and the right one is configured as a lattice structure.

At this point, it is important to know that until recently shell/lattice designs were practically impossible to produce. This, however, is now changing very rapidly by the development of new additive manufacturing production technologies, also termed 3D printing technologies. Interestingly, it is important to know that 3D printed materials are typically rather sensitive to fatigue crack initiation. This makes the engagement of optimization procedures, which remove stress concentrations almost an absolute necessity. Otherwise, the lifespan of such parts may suffer substantially. 
To illustrate the differences between the conventional and configuration-based optimization, let consider a simple part - a specimen to undergo a 3-point bending test (see Fig. 2). The mid-region (depicted in blue) is considered to be the optimization domain. By running the conventional optimization, one gets the optimal design behaving excellently under the precisely defined loading case. On the other hand, configuration-based optimization engages configuration tools before running optimization. This step generates some initial shell/lattice design and the corresponding configuration limits that prevent the optimization to end up at the conventional optimum. Once the design space is configured, optimization may redistribute the material only within the configuration limits. This process greatly reduces the stress concentrations and stress levels but preserves the shell/lattice configuration. In the mathematical sense, the obtained result is less optimal than the conventional optimum. However, it is expected to behave better under variable (not considered) loading situations.
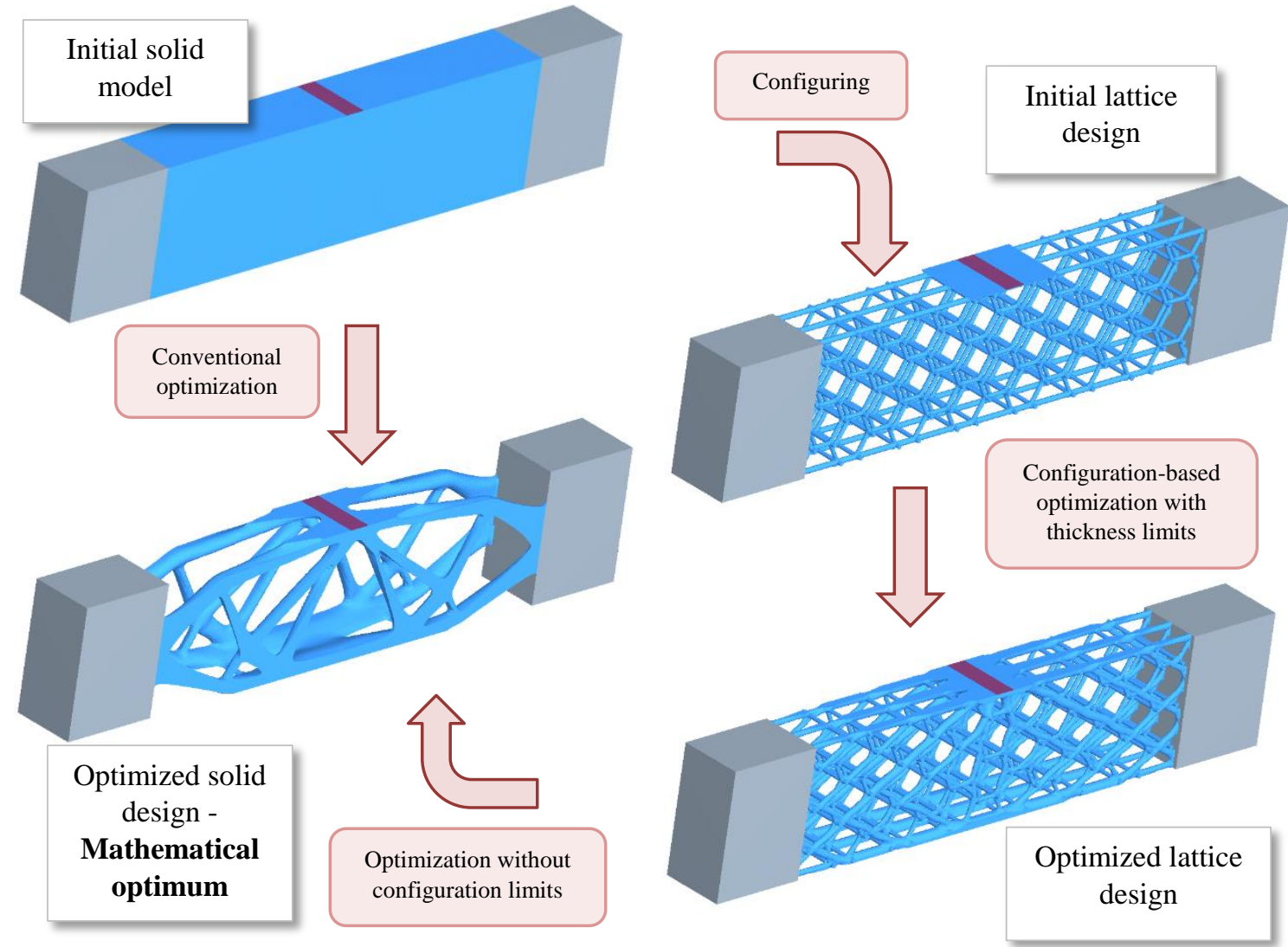

Figure 2: Conventional versus configuration-based optimization.

Lastly, it might be worth mentioning an interesting observation which confirms that the shell/lattice design is suboptimal in the mathematical sense. Namely, if one takes the optimized shell/lattice design, removes the configuration limits, and runs the optimizer, the process should ultimately yield the conventional optimum (see Fig. 2). This can easily be confirmed numerically, if only the optimizer does not get trapped into a local optimum.

\section{NUMERICAL EXAMPLE}

To illustrate and study the features of both optimization approaches a very basic numerical example was selected: a rectangular prismatic specimen undergoing a 3-point bending test. The reason for this choice is the fact that such an example can easily be investigated also experimentally, which was planned in this work. Design configuring and optimization was 
done by CAESS ProTOp [14]; independent numerical response verification was done by SIMULIA Abaqus [15].

\subsection{Numerical model}

The specimen of $125 \times 25 \times 12.5 \mathrm{~mm}$ and the corresponding boundary conditions are shown in Fig. 3. The bending length is $L=100 \mathrm{~mm}$ and the loading force is $F=250 \mathrm{~N}$. Material properties are: $E=1700 \mathrm{MPa}$ and $v=0.3$. Small strain theory and linear elasticity were used for numerical analysis and optimization.

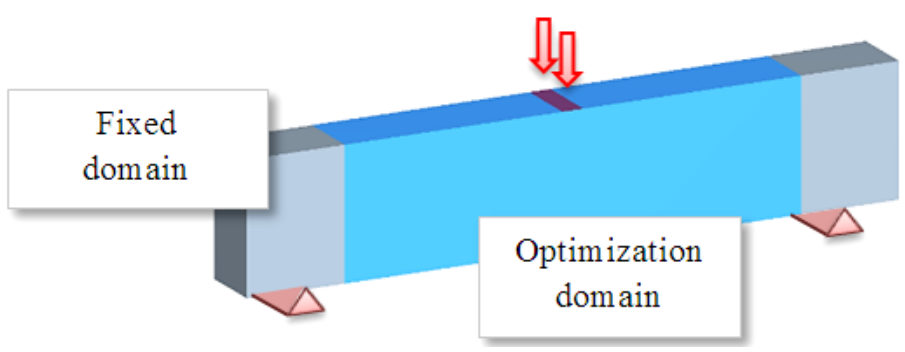

Figure 3: CAD model of the specimen with boundary conditions.

The model consists of two parts: the optimization part in the middle (blue) and fixed domains (grey). Four different configurations, shown in Fig. 4, will be considered to study the effects of different configurations and optimization, see Table I. The volume part (within the optimized region) of all designs is about $12 \%$.
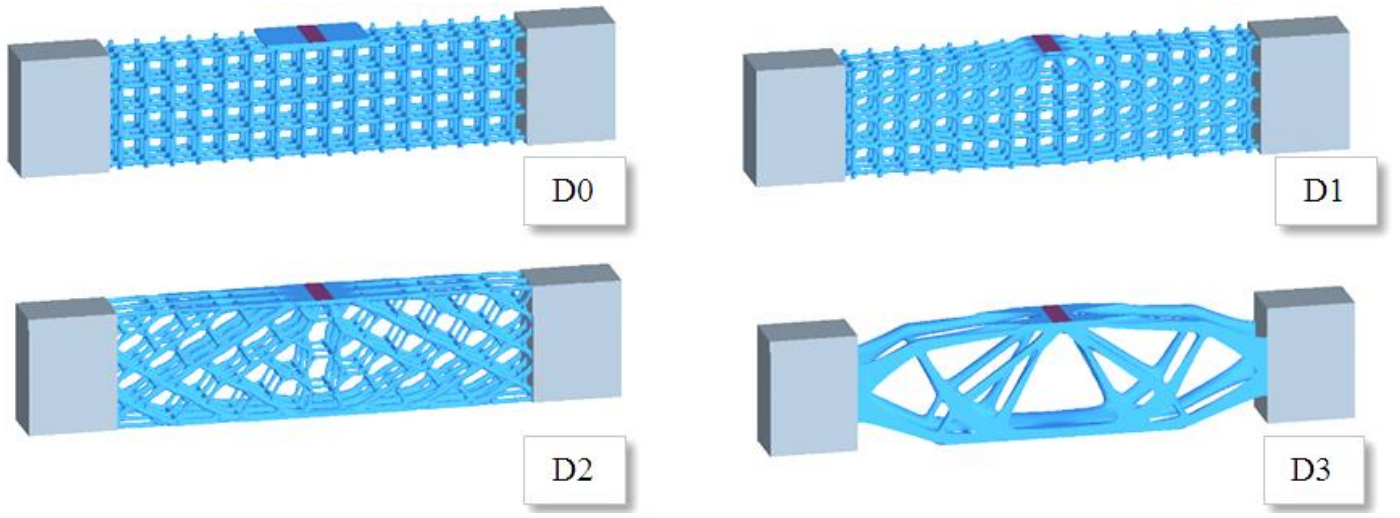

Figure 4: Considered designs: rectangular lattice (D0); optimized rectangular lattice (D1); optimized honeycomb lattice (D2); conventionally optimized structure (D3).

Table I: Considered designs and their properties.

\begin{tabular}{|c|c|c|c|c|}
\hline Design & Configuration & Cell type & Optimized & Notes \\
\hline D0 & Lattice & rectangular & NO & $\begin{array}{c}\text { unfavourable cell } \\
\text { position / orientation }\end{array}$ \\
\hline D1 & Lattice & rectangular & YES & $\begin{array}{c}\text { unfavourable cell } \\
\text { position / orientation }\end{array}$ \\
\hline D2 & Lattice & honeycomb & YES & \\
\hline D3 & (unconstrained - solid) & N/A & YES & \\
\hline
\end{tabular}

\subsection{Numerical results}

According to the theory, the quality of all four designs should be ranked as follows: D0 (the worst), D1, D2, and D3 (the best). The first confirmation of this comes from the computed stress fields (see Fig. 5), where the purple colour indicates stress levels above $80 \mathrm{MPa}$. 


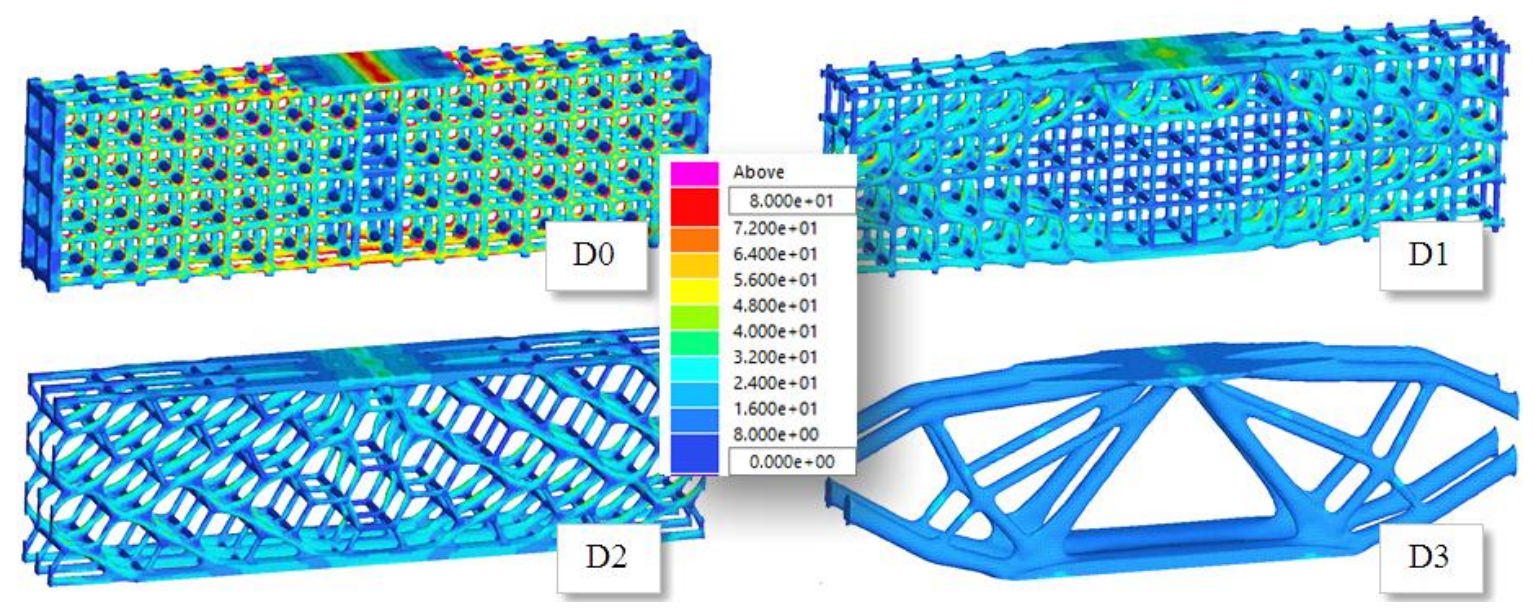

Figure 5: Von Mises stress fields of designs D0, D1, D2, and D3.

One can see that the overall stress levels gradually decrease from design D0 to D3. Numerical results, including approximate maximal stress levels, are listed in Table II. By comparing D0 and D1 one can see that optimization is extremely important - the maximal stresses decrease from about $145 \mathrm{MPa}$ down to about $85 \mathrm{MPa}$ for the same lattice type and orientation. Another important observation is that lattice type and orientation are also very influencing. By comparing D1 and D2 one can see that the stresses decrease from 85 to 43 $\mathrm{MPa}$; this decrease is due to a more favourable lattice type and orientation of design $\mathrm{D} 2$, compared to D1. Finally, from Table II it is also obvious that the structural stiffness (load force divided by maximal displacement) monotonically increases from design D0 to design D3 - exactly as expected from the theoretical point of view.

Table II: Maximal stress levels, maximal displacement, and stiffness of all designs.

\begin{tabular}{|l|c|c|c|c|}
\hline \multicolumn{1}{|c|}{ Design } & D0 & D1 & D2 & D3 \\
\hline Volume part, \% & 12.1 & 11.9 & 12.0 & 12.1 \\
\hline Max stress levels, MPa & $\sim 145$ & $\sim 85$ & $\sim 43$ & $\sim 15$ \\
\hline Max displacement, mm & 8.77 & 4.27 & 2.07 & 1.54 \\
\hline Stiffness, N/mm & 28.5 & 58.6 & 120.5 & 162.8 \\
\hline
\end{tabular}

To illustrate the importance of the optimization, it might be worth to take a look at some design detail and the corresponding stresses (Fig. 6).

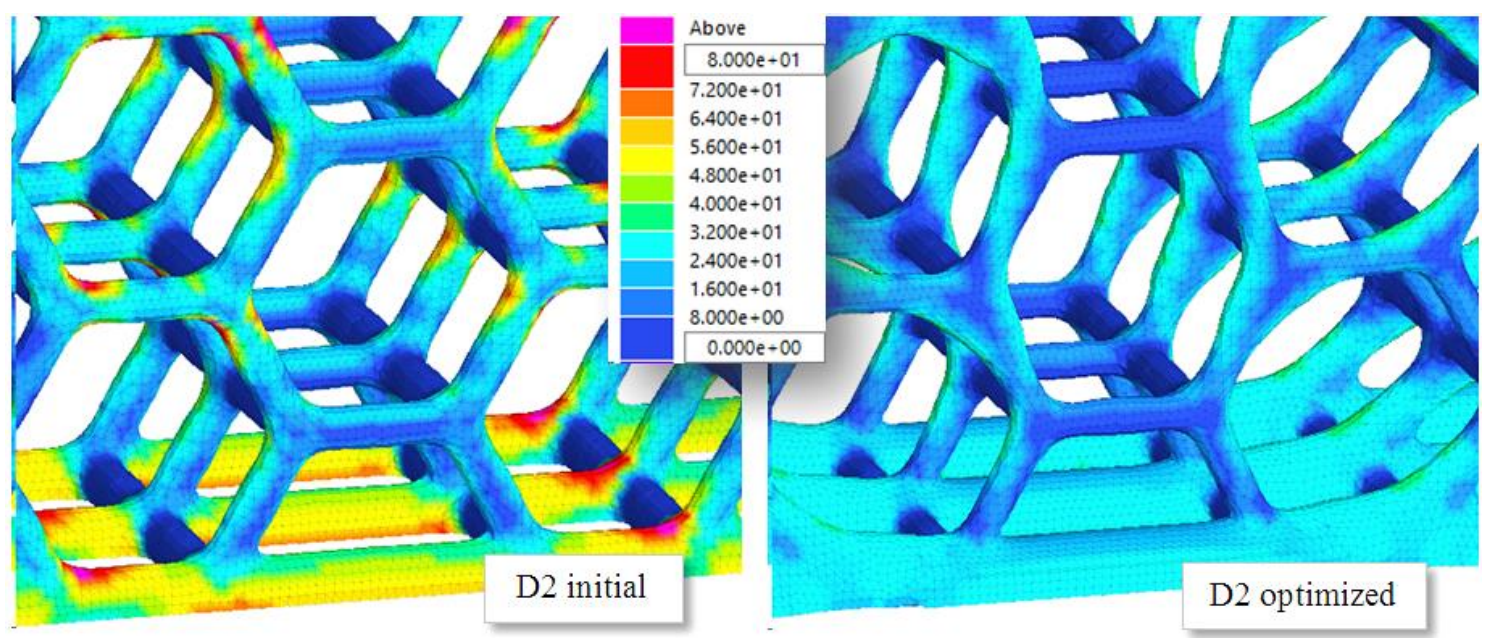

Figure 6: Design detail with a von Mises stress comparison: initial honeycomb lattice structure against the optimized (D2) one. 
Fig. 6 compares two corresponding design details: one of the initial (not optimized) honeycomb lattice structure against the optimized version (design D2). One can clearly see the stress concentrations are vanishing while the max stress levels are reduced from about 95 to about $43 \mathrm{MPa}$.

\section{COMPARISON TO EXPERIMENTAL DATA}

In order to verify the numerical results obtained for the designs D0, D1, D2, and D3, the structures were 3D printed and submitted to a bending test. The tested parts were made by the 3D printer EOS Formiga P100 from the Fine Polyamide PA 2200 for EOSINT P material. The properties of this material are: $E=1700 \pm 150 \mathrm{MPa}, v=0.3$, and the tensile strength is $45 \pm 3 \mathrm{MPa}$.

The three-point bending tests were conducted on the universal hydraulic testing machine INSTRON 8500 (see Fig. 7). The tests performed at room temperature of $+23{ }^{\circ} \mathrm{C}$ were displacement-controlled and the velocity of the displacement was $2 \mathrm{~mm} / \mathrm{min}$.

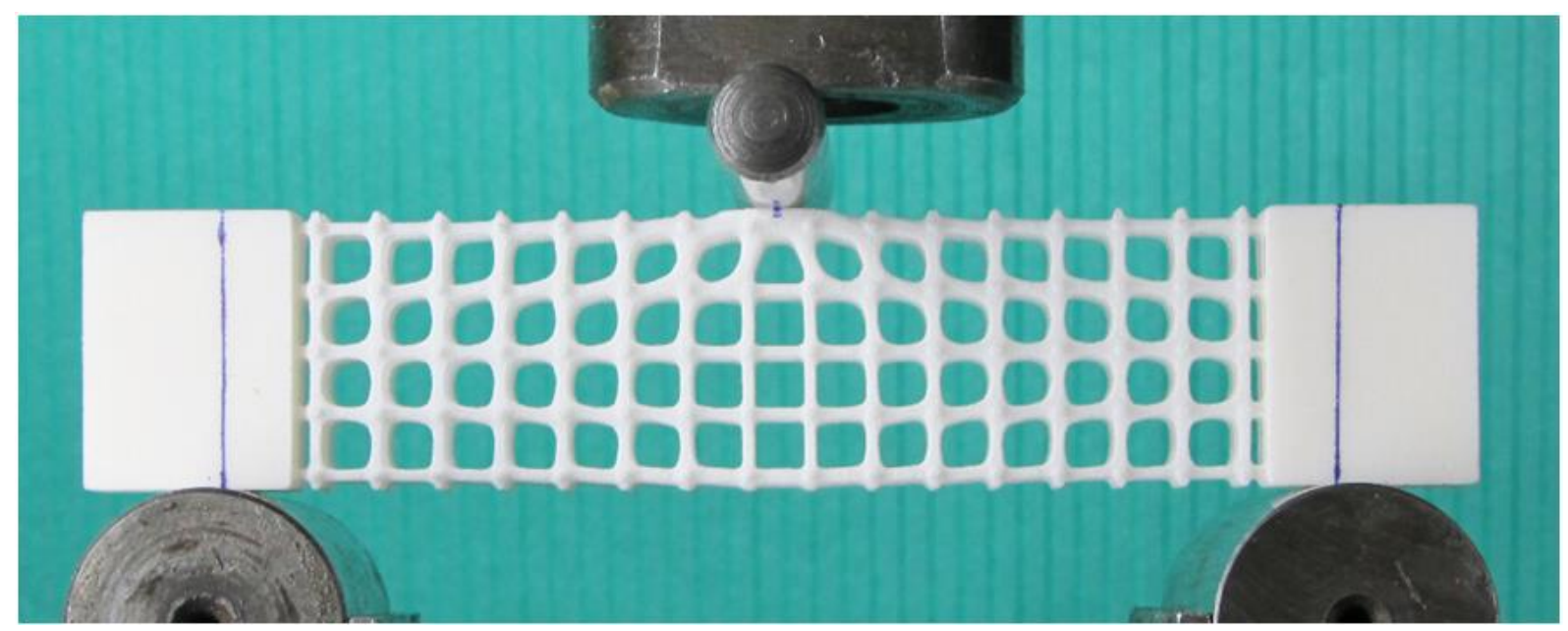

Figure 7: The 3-point bending test of design D1.

Besides of the experiment, the responses of all four designs were also validated by independent simulations by using SIMULIA Abaqus. In these simulations, displacementcontrolled loading was also used in order to have a better comparison against the experimental data. This comparison is shown in Fig. 8, where the dotted lines indicate possible variations of the numerical results due to the uncertainties related to the elastic modulus of the printing material.

At this point it is worth noting that topology optimization methods operate with fictitious material properties, simulating either near-void or near-full-material situations. This induces numerical error that might be especially amplified when considering lattice structures. Namely, lattice structures are the one with the most material-void interface surfaces where these errors could become significant. Luckily, as this comparison indicates, the actual errors seem to be rather small, since the numerical results compare excellent with the experiment. 


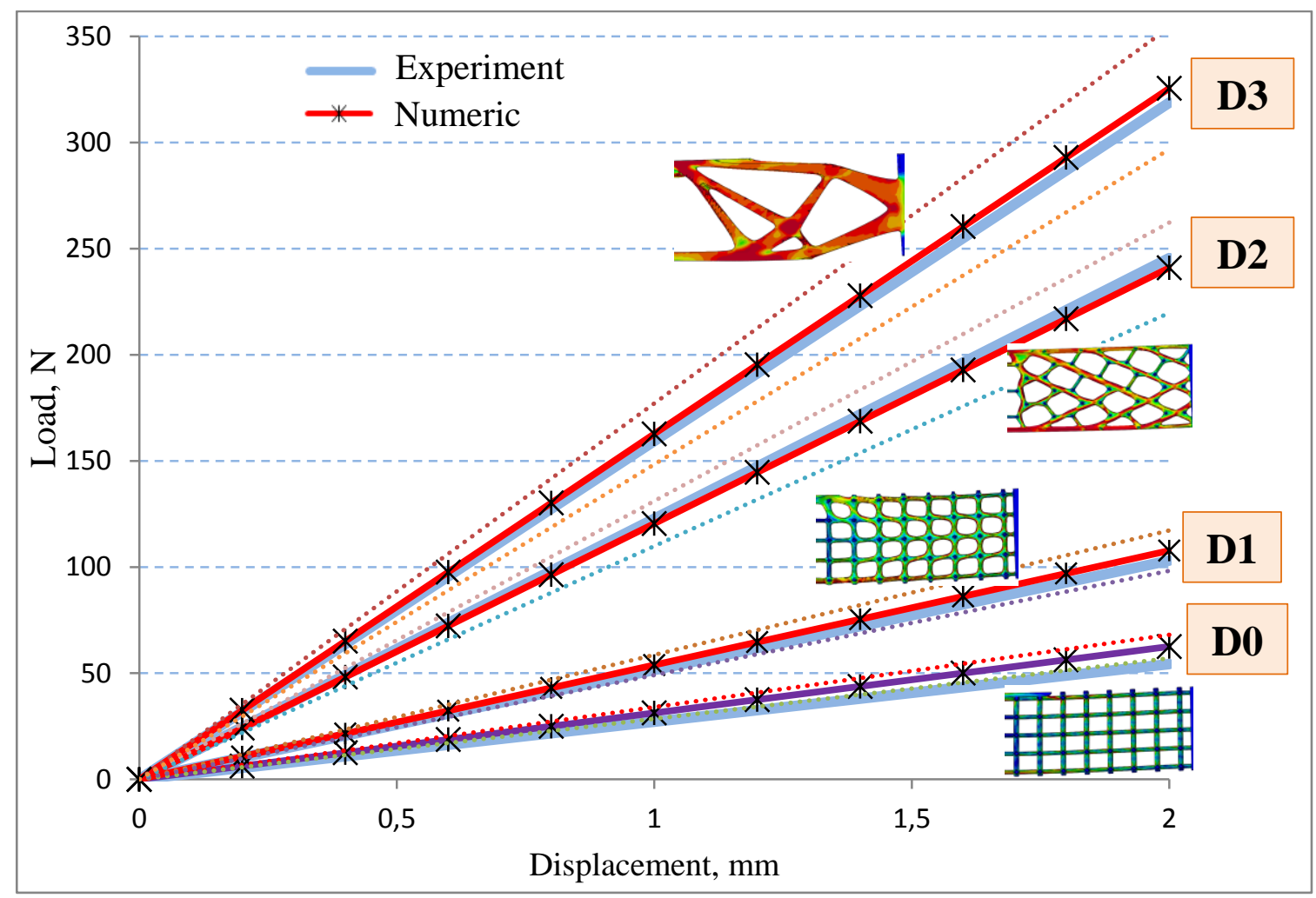

Figure 8: Comparison of experimental and numerical results - displacement-controlled loading.

\section{CONCLUSIONS}

Topology optimization of a solid model adjusts the design to the maximum extent possible to the underlying boundary conditions, i.e., loads and supports. Consequently, the obtained design is excellent for precisely this situation, but might be very sensitive to unexpected variations of these conditions. A challenging question here is how one can get a more robust but still very good design (i.e., in mathematical measures close to the solid optimum).

One of very promising approaches is to engage shell/lattice configuring and optimization. Namely, optimized lattice structures come with the following attractive features:

- They are expected to be much more resistant to variations in load and support conditions than solid optimum designs.

- Problems related to local buckling are expected to be substantially reduced.

- They are expected to be much more resistant to structural failure related to fatigue cracks in the material (fatigue crack of one lattice might be rather irrelevant for the complete structure).

It is important to note, however, that lattice structures typically exhibit stress concentrations, if they are not optimized. Therefore, running topology optimization on a geometrically generated lattice structure should be done by all means. Experience shows that already partly optimized structures exhibit drastically reduced stress concentrations.

Besides of this, the examples provided clearly show that lattice cell type and orientation have a significant influence on the final result. Lattice cells that are shaped and oriented in a favourable manner enable much better final designs.

Finally, it might be worth noting that topology optimization software can deliver reliable results even for designs with a large share of material-void interface surfaces. As the comparison with the experiment revealed, the accuracy of the obtained results is very satisfactory even for a lattice structure. 


\section{REFERENCES}

[1] Rozvany, G. I. N. (2009). A critical review of established methods of structural topology optimization, Structural and Multidisciplinary Optimization, Vol. 37, No. 3, 217-237, doi: $10.1007 / \mathrm{s} 00158-007-0217-0$

[2] Bendsøe, M. P.; Kikuchi, N. (1988). Generating optimal topologies in structural design using a homogenization method, Computer Methods in Applied Mechanics and Engineering, Vol. 71, No. 2, 197-224, doi:10.1016/0045-7825(88)90086-2

[3] Bendsøe, M. P. (1989). Optimal shape design as a material distribution problem, Structural and Multidisciplinary Optimization, Vol. 1, No. 4, 193-202, doi:10.1007/BF01650949

[4] Rozvany, G. I. N. (2001). Aims, scope, methods, history and unified terminology of computeraided topology optimization in structural mechanics, Structural and Multidisciplinary Optimization, Vol. 21, No. 2, 90-108, doi:10.1007/s001580050174

[5] Xie, Y. M.; Steven, G. P. (1993). A simple evolutionary procedure for structural optimization, Computers \& Structures, Vol. 49, No. 5, 885-896, doi:10.1016/0045-7949(93)90035-C

[6] Sethian, J. A.; Wiegmann, A. (2000). Structural boundary design via level set and immersed interface methods, Journal of Computational Physics, Vol. 163, No. 2, 489-528, doi: $10.1006 /$ jcph.2000.6581

[7] Wang, M. Y.; Wang, X.; Guo, D. (2003). A level set method for structural topology optimization, Computer Methods in Applied Mechanics and Engineering, Vol. 192, No. 1-2, 227-246, doi:10.1016/S0045-7825(02)00559-5

[8] Komeili, M.; Menon, C. (2016). Robust design of thermally actuated micro-cantilever using numerical simulations, International Journal of Simulation Modelling, Vol. 15, No. 3, 409-422, doi:10.2507/IJSIMM15(3)2.340

[9] Tadic, B.; Todorovic, P.; Novkinic, B.; Buchmeister, B.; Radenkovic, M.; Budak, I.; Vukelic, D. (2015). Fixture layout design based on a single-surface clamping with local deformation, International Journal of Simulation Modelling, Vol. 14, No. 3, 379-391, doi:10.2507/ IJSIMM14(3)1.280

[10] Hentati, T.; Dammak, F.; Fakhfakh, T.; Haddar, M. (2005). A finite element development for ball bearing nonlinear stiffness modelization, International Journal of Simulation Modelling, Vol. 4, No. 3, 118-128, doi:10.2507/IJSIMM04(3)2.046

[11] Xie, H. L.; Liu, Z. B.; Yang, J. Y.; Sheng, Z. Q.; Xu, Z. W. (2016). Modelling of magnetorheological damper for intelligent bionic leg and simulation of knee joint movement control, International Journal of Simulation Modelling, Vol. 15, No. 1, 144-156, doi:10.2507/IJSIMM15(1)CO2

[12] Esteves, F. R.; Carvalho, T. A.; Pouzada, A. S.; Martins, C. I. (2013). The influence of processing on the aesthetic, morphological and mechanical properties of structural foam mouldings of high- impact polystyrene, Strojniski vestnik - Journal of Mechanical Engineering, Vol. 59, No. 11, 637-645, doi:10.5545/sv-jme.2013.997

[13] Harl, B.; Predan, J.; Kegl, M.; Gubeljak, N. (2015). Design of structural parts by using modern simulation procedures, Zupan, D.; Hozjan, T. (Eds.), Proceedings - Zbornik del, Kuhljevi dnevi 2015 (in Slovenian), doi:10.13140/RG.2.1.3583.0882

[14] CAESS ProTOp, from http://www.caess.eu/site/Software-ProTop.html, accessed on 07-05-2016

[15] SIMULIA Abaqus, from http://www.3ds.com/products-services/simulia/products/abaqus/, accessed on 07-05-2016 